\title{
Characterization of the Darboux Point for Particular Classes of Problems ${ }^{1}$
}

\author{
P. M. MEREAU ${ }^{2}$ AND W. F. Powers ${ }^{3}$ \\ Communicated by J. V. Breakwell
}

\begin{abstract}
A minimal sufficient condition for global optimality involving the Darboux point, analogous to the minimal sufficient condition of local optimality involving the conjugate point, is presented. The Datboux point is then characterized for optimal control problems with linear dynamics, cost functionals with a general terminal state term and an integrand quadratic in the state and control, and general terminal conditions. The Darboux point is shown to be the supremum of a sequence of conjugate points. If the terminal state term is quadratic, along with a scalar quadratic boundary condition, then the Darboux point is also the time at which the Riccati matrix becomes unbounded, giving a characterization of the unboundedness of the Riccati matrix at points which are not in general conjugate points.
\end{abstract}

Key Words. Calculus of variations, optimal control, global sufficient conditions, Darboux point, conjugate point.

\section{Introduction and Preliminaries}

The concept of a Darboux point was formalized in Ref. 1, and several related properties have been proved for a general class of problems (Refs. 1-2). The Darboux point appears as an extension to global optimality of the conjugate point concept, in the sense that it allows a minimal sufficient condition for global optimality (i.e., the gap between necessary and sufficient conditions is minimal). The following definition was proposed. ${ }^{4}$

\footnotetext{
${ }^{1}$ This research was supported by the National Science Foundation under Grant No. GK30115.

${ }^{2}$ Engineer, ADERSA/GERBIOS, Velizy, France.

${ }^{3}$ Professor, Department of Aerospace Engineering, University of Michigan, Ann Arbor, Michigan.

${ }^{4}$ This is Definition 2.1 of Ref. 1.
} 
Definition 1.1. Let $x^{*}(t), t \in\left[t_{0}, t_{f}^{*}\right]$, be a trajectory which satisfies the minimum principle. A point $t_{D} \in\left[t_{0}, t_{f}^{*}\right)$ is called a Darboux point if the following conditions are satisfied ${ }^{5}$ :

(i) for all $t_{2} \in\left(t_{D}, t_{f}^{*}\right)$, there does not exist an admissible trajectory $x(t), t \in\left[t_{D}, t_{f}\right]$, with $x\left(t_{2}\right)=x^{*}\left(t_{2}\right)$, giving a smaller value to the cost functional between $t_{2}$ and $t_{f}$ than $x^{*}(t)$ between $t_{2}$ and $t_{f}^{*}$;

(ii) for all $t_{1} \in\left[t_{0}, t_{D}\right)$, there exists an admissible trajectory $\hat{x}(t), t \in$ $\left[t_{2}, \hat{f}_{f}\right]$, with $x\left(t_{1}\right)=x^{*}\left(t_{1}\right)$, giving a smaller value to the cost functional between $t_{1}$ and $\hat{t}_{f}$ than $x^{*}(t)$ between $t_{1}$ and $t_{f}^{*}$.

It follows that a Darboux point on $\left(t_{0}, t_{f}\right)$ indicates nonglobal optimality. One can make a distinction between two types of Darboux points: Type-1 Darboux points, indicating only the loss of global optimality; and Type-2 Darboux points, indicating also the loss of the existence of a solution to the problem (see Refs. 1-2 for details and examples).

In this paper, a method is proposed to characterize Darboux points for problems with linear dynamics, quadratic cost functionals, and analytic terminal constraints. A sufficient condition for global optimality will be presented in Section 2, and several formulas for the Darboux point will be obtained in Sections 3-6 for various classes of terminal conditions. Examples will be given to illustrate pertinent aspects of the Darboux point and its characterizations.

We shall consider problems of the following form: minimize the functional

$$
J=g\left(x_{f}\right)+\frac{1}{2} \int_{t_{0}}^{t_{f}}\left[x^{T} C(t) x+u^{T} E(t) u\right] d t
$$

subject to

$$
\begin{aligned}
\dot{x} & =A(t) x+B(t) u, \quad t \in\left[t_{0}, t_{f}\right], \\
x\left(t_{0}\right) & =x_{0}, \\
\psi\left(x_{f}\right) & =0,
\end{aligned}
$$

where $\quad x \in R^{n}, u \in R^{m}, m \leq n, \psi \in R^{p}, p \leq n, t_{0}$ and $t_{f}$ are prescribed, $A(t), B(t), C(t)$, and $E(t)$ are continuous matrices on $\left[t_{0}, t_{f}\right]$ of dimensions consistent with the dimensions of $x$ and $u$. The matrices $C(t)$ and $E(t)$ are symmetric. The following assumptions are made.

(A.1) The problem is normal (Ref. 3).

(A.2) The matrix $E(t)$ is positive definite on $\left[\mathrm{t}_{0}, t_{f}\right]$ (strengthened Legendre-Clebsch condition).

\footnotetext{
${ }^{5}$ When $t_{D}=t_{0}$, condition (ii) can be verified only if the trajectory $x^{*}(t)$ can be extended on a small interval $\left[t_{0}-\epsilon, t_{0}\right)$.
} 
(A.3) The system $(A, B)$ is completely controllable, i.e., the matrix

$$
W\left(t_{1}, t_{2}\right)=\int_{t_{1}}^{t_{2}} \phi\left(t_{1}, t\right) B(t) B^{T}(t) \phi\left(t_{1}, t\right) d t
$$

is positive definite for all $t_{1}, t_{2}$, with $t_{0} \leq t_{1}<t_{2} \leq t_{f}$, where $\phi\left(t, t_{0}\right)$ is the transition matrix of $\dot{x}=A(t) x$.

(A.4) The functions $g(\cdot)$ and $\psi_{j}(\cdot), j=1, \ldots, p$, are analytic on the terminal set $\theta \stackrel{\Delta}{=}\left\{x_{f}: \psi\left(x_{f}\right)=0\right\}$.

Because of Assumption (A.2), there is no loss of generality in assuming no mixed term in the integrand of (1) (see Ref. 4).

First-order necessary conditions for an admissible pair $(u, x)$ [i.e., constraints (2), (3), (4) are satisfied] to be optimal are that there exist an absolutely continuous function $\lambda(t) \in R^{n}, t \in\left[t_{0}, t_{f}\right]$, and a constant $\nu \in R^{p}$ such that

$$
\begin{gathered}
\dot{\lambda}(t)=-C(t) x(t)-A^{T}(t) \lambda(t), \quad t \in\left[t_{0}, t_{f}\right], \\
E(t) u(t)+B^{T}(t) \lambda(t)=0, \\
\lambda\left(t_{f}\right)=\left[d g\left(x_{f}\right) / d x_{f}\right]^{T}+\left[d \psi\left(x_{f}\right) / d x_{f}\right]^{T} \nu .
\end{gathered}
$$

Suppose that we have an extremal pair $\left(u^{*}(t), x^{*}(t)\right), t \in\left[t_{0}, t_{f}\right]$, and let $\lambda^{*}(t), t \in\left[t_{0}, t_{f}\right]$, and $\nu^{*}$ be the associated multiplier function and constant Lagrange multiplier. The definition of a Darboux point given above implies the following sufficient condition for global optimality.

Theorem 1.1. Sufficient Condition. An admissible pair $\left(u^{*}(t), x^{*}(t)\right)$, $t \in\left[t_{0}, t_{f}\right]$, is a globally optimal pair if

(i) the first-order necessary conditions are satisfied;

(ii) $E(t)$ is positive definite on $\left[t_{0}, t_{f}\right]$;

(iii) there is no Darboux point on $x^{*}(t)$ for $t \in\left[t_{0}, t_{f}\right)$.

This sufficient condition is minimal, since the only difference with necessary conditions lies in (ii), where $E(t)$ is required to be positive semidefinite on $\left[t_{0}, t_{f}\right]$, and in (iii), where a Darboux point is not allowed on $\left(t_{0}, t_{f}\right)$ for global optimality. In this paper, we shall develop a characterization of the Darboux point which allows a test of (iii).

Define the $n \times n$ matrix $F(q)$ and the $p \times n$ matrix $T(q)$ as

$$
\begin{gathered}
F(q)=2 \sum_{k=2}^{\infty}(1 / k !)\left(d^{k} / d x_{f}^{k}\right)\left[G\left(x_{f}^{*}, \nu^{*}\right)\right]\left(q-x_{f}^{*}\right)^{k-2}, \\
T^{T}(q)=\left[T_{1}(q), \ldots, T_{p}(q)\right], \\
T_{j}(q)=\sum_{k=1}^{\infty}(1 / k !)\left(d^{k} / d x_{f}^{k}\right)\left[\psi_{j}\left(x_{f}^{*}\right)\right]\left(q-x_{f}^{*}\right)^{k-1}, \quad j=1, \ldots, p,
\end{gathered}
$$


in tensor notation, $x_{f}^{*}=x^{*}\left(t_{f}\right), q$ is a constant $n$-dimensional vector with values in the terminal set $\theta$, and $G\left(x_{f}, \nu^{*}\right)$ is defined as

$$
G\left(x_{f}, \nu^{*}\right) \triangleq g\left(x_{f}\right)+\nu^{* T} \psi\left(x_{f}\right) .
$$

Note that, for any $q \in \theta$, Assumption (A.4) and Eqs. (8)-(10) imply that

$$
\begin{gathered}
G\left(q, \nu^{*}\right)=G\left(x_{f}^{*}, \nu^{*}\right)+\left(d / d x_{f}\right) G\left(x_{f}^{*}, \nu^{*}\right)\left(q-x_{f}^{*}\right)+\frac{1}{2}\left(q-x_{f}^{*}\right)^{T} F(q)\left(q-x_{f}^{*}\right), \\
\psi(q)=\psi\left(x_{f}^{*}\right)+T(q)\left(q-x_{f}^{*}\right) .
\end{gathered}
$$

Now, let the matrices $S(t, q), R(t, q)$, and $Q(t, q)$ be the solutions of

$$
\begin{aligned}
& \dot{S}=-S A(t)-A^{T}(t) S-C(t)+S B(t) E^{-1}(t) B^{T}(t) S, \quad S\left(t_{f}, q\right)=F(q), \\
& \dot{R}=-\left[A^{T}(t)-S(t, q) B(t) E^{-1}(t) B^{T}(t)\right] R, \quad R\left(t_{f}, q\right)=T^{T}(q) \\
& \dot{Q}=R^{T}(t, q) B(t) E^{-1}(t) B^{T}(t) R(t, q), \quad Q\left(t_{f}, q\right)=0
\end{aligned}
$$

and define

$$
M(t, q) \triangleq S(t, q)-R(t, q) Q^{-1}(t, q) R^{T}(t, q) .
$$

Let $t_{s q}$ and $t_{c q}$ designate the first times when the matrices $S(t, q)$ and $M(t, q)$ become infinite ${ }^{6}$ when integrated backward from $t_{f}\left(t_{s q}\right.$ and $t_{c q}$ may be equal to $-\infty)$. Notice that, when $q=x^{*}\left(t_{f}\right)$,

$$
F(q)=\left(d^{2} / d x_{f}^{2}\right)\left[G\left(x_{f}^{*}, \nu^{*}\right)\right], \quad T(q)=\left(d / d x_{f}\right) \psi\left(x_{f}^{*}\right),
$$

and the matrices $S(t, q), R(t, q), Q(t, q)$ reduce to the usual $S, R, Q$ matrices used to test for conjugate points (see Ref. 5, Chapter 6 ). Thus, the first backward conjugate point on $x^{*}(t)$ is given by $t_{c q^{*}}$, where $q^{*}=x^{*}\left(t_{f}\right)$.

We shall close this section by presenting a property which will be used later.

Lemma 1.1. Given an extremal trajectory $x^{*}(t), t \in\left[t_{0}, t_{f}\right]$, if there exists another extremal trajectory going through $x^{*}\left(t_{1}\right)$ for some $t_{1} \in\left(t_{0}, t_{f}\right)$ and giving the same value to the cost functional between $t_{1}$ and $t_{f}$ as $x^{*}$ between $t_{1}$ and $t_{f}$, then the trajectory $x^{*}$ is not globally optimal on $\left[t_{0}, t_{f}\right]$.

A proof of this lemma is given in Ref. 2, Appendix D.

\section{Sufficient Condition for Global Optimality}

Using the assumptions and notation defined in Section 1, we have the following property.

\footnotetext{
${ }^{6}$ A matrix is infinite if at least one of its elements is infinite.
} 
Lemma 2.1. If, for a given $q \in \theta$, the matrix $M(t, q)$ is finite on $\left[t_{0}, t_{f}\right)$, then the trajectory $x^{*}(t), t \in\left[t_{0}, t_{f}\right]$, gives a strictly smaller value to the cost functional than any other admissible trajectory terminating at $q$ [i.e., $\left.x\left(t_{f}\right)=q\right]$.

The proof of this lemma is given in Appendix A. A consequence of Lemma 2.1 is the following sufficient condition for global optimality.

Theorem 2.1. Sufficient Condition. An admissible pair $\left(u^{*}(t), x^{*}(t)\right)$, $t \in\left[t_{0}, t_{f}\right]$, is a proper globally optimal pair if

(i) the first-order necessary conditions are satisfied;

(ii) $E(t)$ is positive definite on $\left[t_{0}, t_{f}\right]$;

(iii) $t_{0}>\sup _{q \in \theta} t_{c q}$.

Proof. Condition (iii) implies that $M\left(t_{2} q\right)$ is finite on $\left[t_{0}, t_{f}\right)$ for all $q \in \theta$, and Theorem 2.1 follows from Lemma 2.1.

We will see in the following sections that the sufficient condition of Theorem 2.1 is equivalent to the minimal sufficient condition of Theorem 1.1 under certain conditions.

\section{Case of Linear Terminal Constraints}

Suppose that $g\left(x_{f}\right)$ and $\psi\left(x_{f}\right)$ have the following form:

$$
\begin{aligned}
& g\left(x_{f}\right)=\frac{1}{2} x_{f}^{T} F x_{f}, \\
& \psi\left(x_{f}\right)=T x_{f}+\psi_{0},
\end{aligned}
$$

where $F$ is a symmetric $n \times n$ matrix, $T$ is a $p \times n$ matrix of maximal rank, and $\psi_{0}$ is a $p$-dimensional constant vector. The problem defined in Section 1 is then a linear-quadratic problem (LQP), and the properties of a conjugate point (Ref. 4), together with Definition 1.1 of a Darboux point, imply the following theorem.

Theorem 3.1. For a linear-quadratic problem, a Darboux point on an extremal trajectory occurs at the first backward conjugate point.

Note that the matrices $F(q)$ and $T(q)$ of Section 1 reduce respectively to $F$ and $T$, and $M(t, q)$ is independent of $q$. Thus, we have

$$
\sup _{q \oplus \theta} t_{c q}=t_{c}=t_{D}
$$

a Darboux point. This result will be generalized in the next section. 


\section{Case of $n-1$ Terminal Constraints}

Suppose that $p=n-\omega$ and $g\left(x_{f}\right), \psi_{i}\left(x_{f}\right), j=1, \ldots, n-1$, are arbitrary analytic functions on $\theta$. We make the following assumption:

(A.5) $\operatorname{rank}[T(q)]=p$ for all $q$ in $\theta$.

We will now show that $\sup _{q \in \theta} t_{c q}$ is a Darboux point on $x^{*}$. Theorem 2.1 indicates that condition (i) of Definition 1.1 is satisfied, and it remains to be shown that condition (ii) is also satisfied. Given $q \in \theta$, consider the following (LQP): minimize

$$
I_{q}=\frac{1}{2} y_{f}^{T} F(q) y_{f}+\frac{1}{2} \int_{t_{0}}^{t_{f}}\left[y^{T} C(t) y+v^{T} E(t) v\right] d t,
$$

subject to

$$
\dot{y}=A(t) y+B(t) v, \quad y\left(t_{0}\right)=y_{0} \quad T(q) y_{f}=0 .
$$

This problem is denoted by the abbreviation (LQP) $)_{q}$.

We have the following lemma.

Lemma 4.1. If, for a given $q \in \theta$, the matrix $M(t, q)$ becomes infinite at $t_{c q} \in\left(t_{0}, t_{f}\right)$, then there exists an extremal trajectory of $(\mathrm{LQP})_{q}$ going from $y=0$ at $t=t_{c q}$ to $q-x^{*}\left(t_{f}\right)$ at $t=t_{f}$.

Proof. Obviously, $t_{c q}$ is a conjugate point of $(\mathrm{LQP})_{q}$ on the trajectory $y^{*}=0$. It can be shown (Ref. 2, Theorem 3.1) that there exists at least a one-parameter family of extemal trajectories $y_{\alpha}(t), t \in\left[t_{c q}, t_{f}\right]$, of $(\mathrm{LQP})_{q}$ going through $y^{*}\left(t_{c q}\right)=0$ at $t=t_{c q}$. Now, because of Assumption (A.5), the terminal set

$$
\theta_{q} \triangleq\left\{y_{f}: T(q) y_{f}=0\right\}
$$

of $(\mathrm{LQP})_{q}$ is an $(n-p)$-dimensional hyperplane in $R^{n}$; since $p=$ $n-1, q-x^{*}\left(t_{f}\right)$, and $y^{*}\left(t_{f}\right)=0$ are in $\theta_{q}$, this set reduces to the line $\left(0, q-x_{f}^{*}\right)$ in $R^{n}$. Thus, the vectors $y_{\alpha}\left(t_{f}\right)-y^{*}\left(t_{f}\right)$ and $\left(q-x_{f}^{*}\right)-y^{*}\left(t_{f}\right)$ have the same direction and $\alpha$, which is proportional to the magnitude of $y_{\alpha}\left(t_{f}\right)-y^{*}\left(t_{f}\right)$ (see Ref. 2, proof of Theorem 3.1), can always be chosen, say $\alpha=\bar{\alpha}$, so that

$$
y_{\bar{\alpha}}\left(t_{f}\right)=q-x_{f}^{*} .
$$

The trajectory

$$
x_{q}(t) \triangleq y_{\dot{\alpha}}(t)+x^{*}(t)
$$

where $y_{\bar{\alpha}}$ has been defined above, is such that $x_{q}\left(t_{f}\right)=q$ and is admissible on $\left[t_{c q}, t_{f}\right]$ for the original problem, since both $y_{\bar{\alpha}}(t)$ and $x^{*}(t)$ satisfy $(2)$ and $q$ satisfies (4). We have, furthermore, the following lemma. 
Lemma 4.2. For a given $q \in \theta$, the trajectory $x_{q}(t)$ defined above gives the same value to the cost functional $J$ (of the original problem) between $t_{c q}$ and $t_{f}$ as $x^{*}$ between $t_{c q}$ and $t_{f}$.

The proof is given in Appendix A.

Now, we can show that $x^{*}$ is no longer globally optimal if one of the matrices $M(t, q)$ is infinite on $\left(t_{0}, t_{f}\right)$.

Theorem 4.1. If there exists $q \in \theta$ such that $t_{c q} \in\left(t_{0}, t_{f}\right)$, then the trajectory $x^{*}(t)$ is not globally optimal on $\left[t_{0}, t_{f}\right]$.

Proof. If the trajectory $x_{q}(t)$ (defined before Lemma 4.2) is extremal on $\left[t_{c q}, t_{f}\right]$, then the result follows from Lemmas 4.2 and 1.1. If $x_{q}$ is not extremal on $\left[t_{c q}, t_{f}\right]$, it is certainly not globally optimal from $\left(x^{*}\left(t_{c q}\right), t_{c q}\right)$, and one can find an admissible trajectory from $\left(x^{*}\left(t_{c q}\right), t_{c q}\right)$ ) giving a smaller value to the cost functional than $x_{q}$. Then, Theorem 4.1 follows from Lemma 4.2 .

Finally, Theorems 2.1, 4.1, and Definition 1.1 imply the following theorem.

Theorem 4.2. For the class of problems defined in Section 1 with $p=n-1$ terminal constraints, a Darboux point on the trajectory $x^{*}$ occuts at

$$
t_{D}=\sup _{q \in \theta} t_{c q}
$$

This theorem implies that the sufficient condition of Theorem 2.1 is equivalent to the minimal sufficient condition of Theorem 1.1.

Example 4.1. Consider the case when

$$
\begin{gathered}
n=m=2, \quad t_{0}=0, \quad t_{f}=1, \\
A(t)=C(t)=\left[\begin{array}{ll}
0 & 0 \\
0 & 0
\end{array}\right], \quad B(t)=E(t)=\left[\begin{array}{ll}
1 & 0 \\
0 & 1
\end{array}\right], \\
x_{0}=\left[\begin{array}{l}
0 \\
1
\end{array}\right], \quad g\left(x_{f}\right)=0, \quad \psi\left(x_{f}\right)=x_{2 f}^{3}-x_{2 f}+x_{1 f}-1
\end{gathered}
$$

in Eqs. (1)-(4). Actually, this example is a minimum distance problem from the point $x_{0}$ to the curve

$$
\psi\left(x_{f}\right)=0
$$

An extremal solution is

$$
u^{*}(t)=\left[\begin{array}{l}
1 \\
1
\end{array}\right], \quad x^{*}(t)\left[\begin{array}{c}
t \\
1-t
\end{array}\right], \quad \lambda^{*}(t)=-u^{*}(t), \quad \nu^{*}=-1,
$$


and it can be shown that, for any

we have

$$
q=\left[\begin{array}{l}
q_{1} \\
q_{2}
\end{array}\right] \in \theta
$$

Then, ${ }^{7}$

$$
\begin{gathered}
S(t, q)=\left[\begin{array}{cc}
0 & 0 \\
0 & -1 /\left(t-1+1 / 2 q_{2}\right)
\end{array}\right] \\
M(t, q)=\frac{1}{(1-t)\left[t-1+\left(1+\left(q_{2}^{2}-1\right)^{2}\right) / 2 q_{2}\right]} \\
\\
\times\left[\begin{array}{cc}
t-1+1 / 2 q_{2} & \left(q_{2}^{2}-1\right) / 2 q_{2} \\
\left(q_{2}^{2}-1\right) / 2 q_{2} & t-1+\left(q_{2}^{2}-1\right)^{2} / 2 q_{2}
\end{array}\right]
\end{gathered}
$$

$$
\begin{gathered}
t_{s q}=1-1 / 2 q_{2}, \quad t_{c q}=1-\left(1+\left(q_{2}^{2}-1\right)^{2}\right) / 2 q_{2}, \\
t_{D}=\sup _{q \in \theta} t_{c q}=\sup _{q_{2} \in[0,+\infty)} t_{c q}=0.523 \text { (given by } q_{2}=1.1 \text { ). }
\end{gathered}
$$

Note that a conjugate point on $x^{*}$ is given by $t_{c q^{*}}$, that is,

$$
t_{c q^{*}}=\left(t_{c q}\right)_{q_{2}=0}=-\infty
$$

which illustrates the possibility of the existence of a Darboux point without the existence of a conjugate point.

Example 4.2. Consider the case when

$$
\begin{gathered}
n=m=1, \quad p=0, \\
A(t)=0, \quad C(t)=-1, \quad B(t)=E(t)=1, \quad t \in\left[t_{0}, \pi\right], \\
x_{0}=0, \quad g\left(x_{f}\right)=\frac{1}{2} x_{f}^{2}\left[\exp \left(-x_{f}\right)-1\right]
\end{gathered}
$$

in Eqs. (1)-(4). An extremal solution is

$$
u^{*}(t)=x^{*}(t)=\lambda^{*}(t)=0, \quad t \in\left[t_{0}, \pi\right]
$$

and we have

$$
F(q)=\exp (-q)-1 \text {. }
$$

This can be obtained by using the relation

$$
\exp \left(-x_{f}\right)=\sum_{k=0}^{\infty}(-1)^{k}\left(x_{f}^{k} / k !\right)
$$

\footnotetext{
$\overline{{ }^{7} \text { By definition, } t_{c q}}<t_{f}=1$ and we can restrict $q_{2}$ to belong to $[0,+\infty)$.
} 
Then,

and

$$
t_{c q}=t_{s q}=\tan ^{-1}\left[\frac{\exp (q)}{(1-\exp (q))}\right]
$$

$$
t_{D}=\sup _{q \in(-\infty,+\infty)} t_{c q}=3 \pi / 4 .
$$

It can be proved that the problem does not have a solution when $t_{0}<3 \pi / 4$. The choice

$$
u=\mathrm{const}=\alpha, \quad x=\alpha\left(t-t_{0}\right)
$$

makes the cost functional as small as desired when $\alpha$ is large enough. This indicates that $t_{D}$ is a Type-2 Darboux point.

We notice that, in Example 4.1, sup $t_{c q}$ was attained (given by $q_{2}=1.1$ ), while it was not attained in Example 4.2. This observation holds in general.

Theorem 4.3. For a Type-1 Darboux point, the supremum of $t_{c q}$ is attained, i.e.,

$$
t_{D}=\max _{q \in \theta} t_{c q} .
$$

Proof. Suppose that $t_{D}$ is a Type-1 Darboux point on $x^{*}$. If $t_{D}$ is a conjugate point on $x^{*}$, then $t_{D}=t_{c q^{*}}$ (see Section 1) and

$$
\sup _{q \in \theta} t_{c q}=t_{D}=t_{c q^{*}}
$$

is attained with

$$
q=q^{*}=x^{*}\left(t_{f}\right)
$$

If $t_{D}$ is not a conjugate point on $x^{*}$, then a property of Type-1 Darboux points (Ref. 1, Theorem 4.1) implies that there exists a trajectory $\bar{x}(t), t \in$ $\left[t_{D}, t_{f}\right]$, distinct from $x^{*}$, globally optimal on $\left[t_{D}, t_{f}\right]$. Let $\bar{q}=\bar{x}\left(t_{f}\right)$. We have

$$
t_{c \bar{q}} \leq \sup _{a \in \theta} t_{c a}=t_{D}
$$

But, if $t_{c \bar{q}}<t_{D}$, Lemma 2.1 implies that $x^{*}$ gives a smaller value to the cost functional $J$ than $\bar{x}$ between $t_{D}$ and $t_{f}$, which is in contradiction with the optimality of $\bar{x}$ between $t_{D}$ and $t_{f}$. Thus, we have

$$
t_{c \bar{q}}=t_{D}=\sup _{q \in \theta} t_{c q}
$$

which completes the proof.

Remark 4.1. For a Type-2 Darboux point, the supremum of $t_{c q}$ may be attained (see Section 3) or may not be attained (see Example 4.2). 


\section{General Case}

Suppose now that $p$ is an arbitrary integer, $0 \leq p \leq n$, that $g\left(x_{f}\right), \psi_{j}\left(x_{f}\right), j=1, \ldots, p$, are arbitrary analytic functions on $\theta$, and that Assumption (A.5) is satisfied. We wish to investigate a generalization of the result of Theorem 4.2. The approach used in Section 4 to show that the trajectory $x^{*}$ is not globally optimal on $\left[t_{0}, t_{f}\right]$ when $t_{0}<t_{D}$ is no longer valid, since Lemma 4.1 is not necessarily true, as shown in the example below.

Example 5.1. Consider the case when

$$
\begin{gathered}
n=m=2, \quad p=0, \\
A(t)=\left[\begin{array}{ll}
0 & 0 \\
0 & 0
\end{array}\right], \quad B(t)=-C(t)=E(t)=\left[\begin{array}{ll}
1 & 0 \\
0 & 1
\end{array}\right], \quad t \in\left[t_{0}, \pi\right], \\
x_{0}=\left[\begin{array}{l}
0 \\
0
\end{array}\right], \quad g\left(x_{f}\right)=x_{1 f}^{3}+\frac{1}{2} x_{1 f}^{4}
\end{gathered}
$$

in Eqs. (1)-(4). An extremal solution is

$$
u^{*}(t)=x^{*}(t)=\lambda^{*}(t)=\left[\begin{array}{l}
0 \\
0
\end{array}\right], \quad t \in\left[t_{0}, \pi\right]
$$

and we have

It follows that

$$
M(t, q)=S(t, q)=\left[\begin{array}{ll}
\frac{\left[q_{1}^{2}+2 q_{1}+\tan t\right]}{\left[1-\left(q_{1}^{2}+2 q_{1}\right) \tan t\right]} & 0 \\
0 & \tan t
\end{array}\right] \quad \text { for all } q=\left[\begin{array}{l}
q_{1} \\
q_{2}
\end{array}\right] \in R^{2}
$$

$$
t_{c q}=\sup \left\{\frac{\pi}{2}, \tan ^{-1}\left[1 /\left(q_{1}^{2}+2 q_{1}\right)\right]\right\}
$$

and

$$
\sup _{q \in \theta}=\sup \left\{\frac{\pi}{2}, \sup _{q_{1} \in(-\infty,+\infty)} \tan ^{-1}\left[1 /\left(q_{1}^{2}+2 q_{1}\right)\right]\right\}=3 \pi / 4 .
$$

It can be shown (Ref. 2, Example 5.3) that the trajectory $y_{\alpha}(t)$ in the proof of Lemma 4.1 is

$$
y_{\alpha}(t)=\left[\begin{array}{c}
-\alpha \sin \left(t-t_{c q}\right) \\
0
\end{array}\right]
$$

It follows that $\alpha$ can be chosen such that $y_{1 \alpha}\left(t_{f}\right)$ has a given value $q_{1}-x_{1}^{*}\left(t_{f}\right)$, but $y_{2 \alpha}\left(t_{f}\right)$ cannot be made to have an arbitrary value $q_{2}-x_{2}^{*}\left(t_{f}\right)$. This shows 
that Lemma 4.1 is not necessarily true when the number $p$ of terminal constraints is arbitrary.

Notice, however, that

$$
\bar{q}=\left[\begin{array}{c}
q_{1} \\
0
\end{array}\right]
$$

is such that

$$
t_{c \bar{q}}=t_{c q}
$$

and that $y_{\bar{\alpha}}\left(t_{f}\right)$, where

$$
\bar{\alpha}=-q_{1} / \sin \left(t_{f}-t_{c q}\right)
$$

satisfies Lemma 4.1 with $q=\bar{q}$. Then, Theorem 4.1 holds with $q=\bar{q}$, and

$$
t_{c \bar{q}}=t_{c q}
$$

implies that $x^{*}$ is not globally optimal when $t_{0}<t_{c q}$. It follows that the Darboux point is still given by $\sup _{q \in \theta} t_{c q}$. This result can be checked by noticing that the problem considered can be separated into two independent problems of the type considered in Section 4 (i.e., $p=n-1$ ).

In general, for a given $q \in \theta$, there does not necessarily exist a $\bar{q}$ as above (i.e., satisfying Lemma 4.1 and $t_{c \bar{q}}=t_{c q}$ ), and we cannot say $a$ priori that the Darboux point is given by $\sup _{q \in \theta} t_{c q}$. For a given $q$ in $\theta$, let $V_{i}^{q}, i=$ $1, \ldots, n-p-1$, be a set of vectors in the $(n-1)$-dimensional subspace of $R^{n}$ orthogonal to the line $\left(q, x_{f}^{*}\right)$, such that the vectors $T_{j}(q), j=1, \ldots, p$, and $V_{i}^{q}$ are linearly independent [this choice is always possible, since the vectors $T_{i}(q)$ are linearly independent, from Assumption $\left.(\mathrm{A} .5)\right]$. Let $(\overline{\mathrm{LQP}})_{q}$ be the problem similar to $(\mathrm{LQP})_{q}$ defined in Section 4 , but with the extra terminal conditions

$$
V_{i}^{q} y_{f}=0, \quad i=1, \ldots, n-p-1,
$$

and define $\bar{t}_{c q}$ as follows.

Definition 5.1. $\bar{t}_{c q}$ is the first backward conjugate point of $(\overline{\mathrm{LQP}})_{q}$ on the trajectory $y^{*}(t)=0$.

We have the following theorem.

Theorem 5.1. For the class of problems defined in Section 1 with an arbitrary number $p, 0 \leq p \leq n$, of terminal constraints, a Darboux point on the trajectory $x^{*}$ occurs at

$$
t_{D}=\sup _{q \in \theta} t_{c q}
$$


Proof. Suppose that

$$
\sup _{q \in \theta} \bar{t}_{c q} \in\left(t_{0}, t_{f}\right)
$$

let

$$
t_{1}=\sup _{q \in \theta} T_{c q}-\epsilon
$$

then, for $\epsilon$ small enough, there exists $\tilde{q} \in \theta$ such that

$$
t_{c \tilde{q}}=t_{1} .
$$

From Lemma 4.1 [note that $(\overline{\mathrm{LQP}})_{q}$ has $n-1$ terminal constraints], there exists an extremal trajectory $\bar{y}(t)$ of $(\overline{\mathrm{LQP}})_{q}$ such that

$$
\bar{y}\left(\bar{t}_{c \bar{q}}\right)=0 \quad \text { and } \bar{y}\left(t_{f}\right)=\bar{q}-x^{*}\left(t_{f}\right) .
$$

The trajectory

$$
\bar{x}(t) \triangleq \bar{y}(t)+x^{*}(t)
$$

is admissible on $\left[t_{1}, t_{f}\right]$ for the original problem, since $\bar{x}(t)$ satisfies (2) and $\bar{x}\left(t_{f}\right)=\bar{q}$ satisfies (4). Then, Lemma 4.2 and Theorem 4.1 hold, and condition (ii) of Definition 1.1 is satisfied.

Now, let

$$
t_{2}>\sup _{q \in \theta} t_{c q}
$$

it follows that the trajectory

$$
y^{*}(t)=0, \quad t \in\left[t_{0}, t_{f}\right],
$$

is proper globally optimal on $\left[t_{2}, t_{f}\right]$ for all $(\overline{\mathrm{LQP}})_{q}, q \in \theta$. Suppose that condition (i) of Definition 1.1 is not satisfied; then, there exists a trajectory $\hat{x}(t)$, admissible on $\left[t_{2}, t_{f}\right]$, of the original problem giving a smaller value to the cost functional between $t_{2}$ and $t_{f}$ than $x^{*}$. The trajectory

$$
\hat{y}(t) \stackrel{\Delta}{=} \hat{x}(t)-x^{*}(t)
$$

is obviously admissible on $\left[t_{2}, t_{f}\right]$ for $(\overline{\mathrm{LQP}})_{\hat{q}}$, where $\hat{q}=\hat{x}\left(t_{f}\right)$, and $\hat{y}$ gives a smaller value to the cost $I_{\hat{q}}$ of $(\overline{\mathrm{LQP}})_{\hat{q}}$ than $y^{*}$ between $t_{2}$ and $t_{f}$, which is a contradiction with $t_{2}>\bar{t}_{c \hat{q}}$. To show that $\hat{y}$ gives a negative value of $I_{\hat{q}}$ consider

$$
\Delta J=G\left(\hat{q}, \nu^{*}\right)-G\left(x^{*}, \nu\right)-\lambda^{* T}\left(t_{f}\right) \Delta x_{f}+\frac{1}{2} \int_{t_{2}}^{t_{f}}\left(\Delta x^{T} C \Delta x+\Delta u^{T} E \Delta u\right) d t,
$$


where $\Delta J$ is the difference between the values of the cost functional given by $\hat{x}(t)$ and $x^{*}(t)$ between $t_{2}$ and $t_{f}$

$$
\Delta x(t) \triangleq \hat{x}(t)-x^{*}(t), \quad \Delta u(t) \triangleq \hat{u}(t)-u^{*}(t)
$$

(see Appendix A, proof of Lemma 4.2). Then,

$$
\Delta J=\frac{1}{2} y_{f}^{T} F(\hat{q}) y_{f}+\frac{1}{2} \int_{t_{2}}^{t_{f}}\left(y^{T} C y+v^{T} E v\right) d t
$$

where

$$
y(t)=\Delta x(t), \quad v(t)=\Delta u(t)
$$

have been used. Therefore, $\Delta J$ is the value between $t_{2}$ and $t_{f}$ of the cost functional $I_{\hat{q}}$ of $(\overline{\mathrm{LQP}})_{\hat{q}}$, and the result follows from $\Delta J<0$. The proof of Theorem 5.1 is thus complete.

Remark 5.1. It can be shown (Ref. 2) that $t_{c q} \leq t_{c q}$ for any $q \in \theta$, and it follows that

$$
t_{D}=\sup _{q \in \theta} t_{c q} \leq \sup _{q \in \theta} t_{c q}
$$

For the problem of Example 5.1, it can be shown that

$$
\bar{t}_{c q}=\sup \left\{\frac{\pi}{2}, \tan ^{-1}\left[\left(q_{1}^{2}+q_{2}^{2}\right) / q_{1}^{2}\left(q_{1}^{2}+2 q_{1}\right)\right]\right\}
$$

and that

$$
t_{D}=\sup _{q \in \theta} t_{c q}=3 \pi / 4=\sup _{q \in \theta} t_{c q}
$$

as expected.

Remark 5.2. $t_{c q}$ is the first time when the matrix $M(t, q)$ becomes infinite when integrated backward. $M(t, q)$ is defined as

$$
\underline{M}(t, q)=S(t, q)-\underline{R}(t, q) Q^{-1}(t, q) \underline{R}^{T}(t, q),
$$

where $S(t, q), \underline{R}(t, q)$, and $Q(t, q)$ are solutions of Eqs. (14)-(16), when $R\left(t_{f}, q\right)$ is replaced by

$$
\underline{R}\left(t_{f}, q\right)=\left[T^{T}(q): V^{q T}\right], \quad V^{q T}=\left[V_{1}^{q}, \ldots, V_{n-p-1}^{q}\right] .
$$

Thus, for an arbitrary number of terminal constraints, a Darboux point $t_{D}$ is less than or equal to $\sup _{q \in \theta} t_{c q}$. A case when

$$
t_{D}=\sup _{q \in \theta} t_{c q}
$$


will be investigated in the next section. The particular structure is such that a Darboux point is easily calculated and, furthermore, is related to the Riccati matrix of the LQP.

\section{Case of One Quadratic Terminal Constraint}

Suppose that $g\left(x_{f}\right)$ and $\psi\left(x_{f}\right)$ have the following form:

$$
\begin{aligned}
& g\left(x_{f}\right)=\frac{1}{2} x_{f}^{T} F_{0} x_{f}, \\
& \psi\left(x_{f}\right)=\frac{1}{2} x_{f}^{T} F_{1} x_{f}+T x_{f}+\psi_{0},
\end{aligned}
$$

where $F_{0}$ and $f_{1}$ are symmetric $n \times n$ matrices, $F_{1} \neq 0$ (i.e., $e^{T} F_{1} e \neq 0$ for all $\left.e \in R^{n}, e \neq 0\right), T$ is a $1 \times n$ vector $(p=1)$, and $\psi_{0}$ is a scalar. The matrices $F(q)$ and $T(q)$ are

$$
F(q)=F_{0}+\nu^{*} F_{1}, \quad \nu^{*} \in R^{1}
$$

and

$$
T(q)=\frac{1}{2}\left(q+x_{f}^{*}\right)^{T} F_{1}+T,
$$

respectively; thus, $S(t, q)$ does not depend on $q$. Accordingly, we shall designate $S(t, q)$ by $S(t)$ and $t_{s q}$ by $t_{s}$.

The major result of this section is that $t_{s}$ is a Darboux point. In this direction, the following corollary of Theorem 2.1 implies that condition (i) of the Darboux point definition (Definition 1.1) is satisfied.

Corollary 6.1. A sufficient condition for an extremal trajectory $x^{*}(t), t \in\left[t_{0}, t_{f}\right]$, to be proper globally optimal is that $t_{0}>t_{s}$.

Proof. It can be shown (Ref. 2, Theorem 3.7) that $t_{s} \geq t_{c q}$ for all $q \in \theta$. Thus,

$$
t_{0}>t_{s} \geq \sup _{q \in \theta} t_{c q}
$$

and the result follows from Theorem 2.1.

We will now follow several steps to show that condition (ii) of Definition 1.1 is satisfied. Let $\Omega\left(t, t_{0}\right)$ be the $2 n \times 2 n$ transition matrix of

$$
\dot{x}=\left[\begin{array}{cc}
A(t) & -B(t) E^{-1}(t) B^{T}(t) \\
-C(t) & -A^{T}(t)
\end{array}\right] x,
$$

and denote by $\Omega_{i j}\left(t, t_{0}\right), i, j=1,2$, the block of $\Omega\left(t, t_{0}\right)$ partitioned into four $n \times n$ blocks. Suppose that $t_{s} \in\left(t_{0}, t_{f}\right)$. Then, there exists a nonzero vector 
$e \in R^{n}$ in the null space of

$$
\bar{S}\left(t_{s}, t_{f}\right)=\Omega_{22}\left(t_{f}, t_{s}\right)-S\left(t_{f}\right) \Omega_{12}\left(t_{f}, t_{s}\right)
$$

(see Ref. 2, Section 3.3). Let $\left(x_{\alpha}(t), \lambda_{\alpha}(t)\right)$ be the solution on $\left[t_{s}, t_{f}\right]$ of Eqs. (2), (5), where (6) and Assumption (A.2) have been used to eliminate $u$, with initial conditions

$$
x\left(t_{s}\right)=x^{*}\left(t_{s}\right), \quad \lambda\left(t_{s}\right)=\lambda^{*}(t)+\alpha e,
$$

where $\alpha$ is a scalar parameter. We have

$$
\begin{aligned}
& x_{\alpha}(t)=\Omega_{11}\left(t, t_{s}\right) x^{*}\left(t_{s}\right)+\Omega_{12}\left(t, t_{s}\right) \lambda\left(t_{s}\right)=x^{*}(t)+\alpha \Omega_{12}\left(t, t_{s}\right) e, \\
& \lambda_{\alpha}(t)=\Omega_{21}\left(t, t_{s}\right) x^{*}\left(t_{s}\right)+\Omega_{22}\left(t, t_{s}\right) \lambda\left(t_{s}\right)=\lambda^{*}(t)+\alpha \Omega_{22}\left(t, t_{s}\right) e,
\end{aligned}
$$

and the following lemma.

Lemma 6.1. If there exists $\alpha \neq 0$ such that $x_{\alpha}\left(t_{f}\right) \in \theta$, then the trajectory $x_{\alpha}(t), t \in\left[t_{s}, t_{f}\right]$, is extremal.

Proof. Since ( $\left(x_{\alpha}, \lambda_{\alpha}\right.$ ) satisfies Eqs. (2), (4), (5), and (6), we only need to show that (7) is satisfied with $\lambda_{\alpha}\left(t_{f}\right)$ and $x_{\alpha}\left(t_{f}\right)$ for some $\nu_{\alpha}$. Using (24), we have

$$
\begin{aligned}
\lambda_{\alpha}\left(t_{f}\right) & -\left(F_{0}+\nu_{\alpha} F_{1}\right) x_{\alpha}\left(t_{f}\right)-\nu_{\alpha} T^{T}=\lambda^{*}\left(t_{f}\right)-\left(F_{0}+\nu^{*} F_{1}\right) x^{*}\left(t_{f}\right)-\nu^{*} T^{T} \\
& -\left(\nu_{\alpha}-\nu^{*}\right)\left\{F_{1}\left[x^{*}\left(t_{f}\right)+\alpha \Omega_{12}\left(t_{f}, t_{s}\right) e\right]+T^{T}\right\} \\
& \left.+\alpha\left[\Omega_{22}\left(t_{f}, t_{s}\right)-\left(F_{0}+\nu^{*} F_{1}\right) \Omega_{12}\left(t_{f}, t_{s}\right)\right]\right] .
\end{aligned}
$$

Then, the satisfaction of (7) with $x_{\alpha}\left(t_{f}\right), \lambda_{\alpha}\left(t_{f}\right), v_{\alpha}$ follows from the definition of $e$, the choice $\nu_{\alpha}=\nu^{*}$, and the satisfaction of (7) with $x^{*}\left(t_{f}\right), \lambda^{*}\left(t_{f}\right), \nu^{*}$.

Next, we examine the existence of an $\alpha \neq 0$ such that $x_{\alpha}\left(t_{f}\right) \in \theta$. This requires, using (24), that

$$
\begin{aligned}
0=\psi[ & \left.x_{\alpha}\left(t_{f}\right)\right]=\frac{1}{2}\left[x^{*}\left(t_{f}\right)+\alpha \Omega_{12}\left(t_{f}, t_{s}\right) e\right]^{T} F_{1}\left[x^{*}\left(t_{f}\right)+\alpha \Omega_{12}\left(t_{f}, t_{s}\right) e\right] \\
& +T\left[x^{*}\left(t_{f}\right)+\alpha \Omega_{12}\left(t_{f}, t_{s}\right) e\right]+\psi_{0}=0,
\end{aligned}
$$

or

$$
\begin{aligned}
0=\psi\left[x^{*}\left(t_{f}\right)\right]+\frac{1}{2} \alpha^{2}\left[\Omega_{12}\left(t_{f}, t_{s}\right) e\right]^{T} F_{1}\left[\Omega_{12}\left(t_{f}, t_{s}\right) e\right] \\
+\alpha\left[x^{* T}\left(t_{f}\right) F_{1}+T\right] \Omega_{12}\left(t_{f}, t_{s}\right) e,
\end{aligned}
$$

with $\psi\left[x^{*}\left(t_{f}\right)\right]=0$. Let $v$ represent the vector $\Omega_{12}\left(t_{f}, t_{s}\right)$. Then, $\alpha$ must be the solution of

$$
\alpha\left(v^{T} F_{1} v\right)+2\left(x_{f}^{* T} F_{1}+T^{T}\right) v=0 .
$$


We have four cases:

Case (i): $v^{T} F_{1} v \neq 0$ and $\left(x_{f}^{* T} F_{1}+T\right) v \neq 0$; then, the solution is

$$
\alpha=-2\left(v^{T} F_{1} v\right)^{-1}\left(x_{f}^{* T} F_{1}+T\right) v \neq 0 .
$$

Case (ii): $v^{T} F_{1} v \neq 0$ and $\left(x_{f}^{* T} F_{1}+T\right) v=0$; then, the only solution is

$$
\alpha=0 \text {. }
$$

Case (iii): $v^{T} F_{1} v=0$ and $\left(x_{f}^{* T} F_{1}+T\right) v \neq 0$; then, the only solution is

$$
\alpha=+\infty \text {. }
$$

Case (iv): $v^{T} F_{1} v=0$ and $\left(x_{f}^{* T} F_{1}+T\right) v=0$; then,

$$
\alpha=\text { any real number. }
$$

Case (iv) corresponds to the case where $\theta$ consists of two hyperplanes ( $\psi$ is the product of two affine functions), one of them containing $v$. Case (iii) corresponds to the case where $x_{\alpha}\left(t_{f}\right)$ is at infinity (for instance, if $\theta$ is a parabola and $v$ is in the direction of the principal axis) and is not realistic, since it means that $x_{\alpha}\left(t_{f}\right)$ is attained in the finite amount of time $t_{f}-t_{s}$ from $x^{*}\left(t_{s}\right)$, with

$$
\left\|x_{\alpha}\left(t_{f}\right)-x^{*}\left(t_{s}\right)\right\|=\infty
$$

However, we will consider this case and view the corresponding trajectory as the limit of $x_{\alpha}(t)$ as $\alpha$ goes to infinity. Case (ii) corresponds to the case where $t_{s}$ is a conjugate point, as shown below.

Lemma 6.2. If there exists a nonzero vector $e$ in the null space of $\bar{S}\left(t_{s,} t_{f}\right)$ such that

$$
\left(x_{f}^{* T} F_{1}+T\right) \Omega_{12}\left(t_{f}, t_{s}\right) e=0,
$$

then $t_{s}$ is a conjugate point on the trajectory $x^{*}$.

\section{Proof. Consider}

$$
V(\tau)=\left[\Omega_{22}\left(\tau, t_{s}\right)-M(\tau) \Omega_{12}\left(\tau, t_{s}\right)\right] e,
$$

where

$$
M(\tau)=M\left(\tau, q=x_{f}^{*}\right)
$$

is the matrix used to test for conjugate points on $x^{*}$ (as noted in Section 1). Using (17) and the definition of $e$, we have

$$
\begin{aligned}
& V(\tau)=\left[\Omega_{22}\left(\tau, t_{s}\right)-S(\tau) \Omega_{12}\left(\tau, t_{s}\right)\right] e+R(\tau) Q^{-1}(\tau) R^{T}(\tau) \Omega_{12}\left(\tau, t_{s}\right) e, \\
& V(\tau)=-R(\tau) Q^{-1}(\tau) R^{T}(\tau) \Omega_{12}\left(\tau, t_{s}\right) e .
\end{aligned}
$$


Define

$$
\rho(\tau) \triangleq R^{T}(\tau) \Omega_{12}\left(\tau, t_{s}\right) e
$$

we have

$$
\begin{aligned}
\dot{\rho}(\tau)= & \dot{R}^{T} \Omega_{12}\left(\tau, t_{s}\right) e+R^{T} \Omega_{12}\left(\tau, t_{s}\right) e \\
= & -R^{T}\left(A-B E^{-1} B^{T} S\right) \Omega_{12}\left(\tau, t_{s}\right) e \\
& +R^{T}\left[A \Omega_{12}\left(\tau, t_{s}\right)-B E^{-1} B^{T} \Omega_{22}\left(\tau, t_{s}\right)\right] e \\
= & -R^{T} B E^{-1} B^{T}\left[\Omega_{22}\left(\tau, t_{s}\right)-S(\tau) \Omega_{12}\left(\tau, t_{s}\right)\right] e=0,
\end{aligned}
$$

where the expression for

$$
\dot{\Omega}\left(\tau, t_{s}\right)=(\partial / \partial \tau) \Omega\left(\tau, t_{s}\right)
$$

is easily derived from the definition of $\Omega\left(t, t_{0}\right)$. Thus, we have

$$
\rho(\tau)=\rho\left(t_{f}\right) \quad \text { for all } \tau \in\left(t_{s}, t_{f}\right]
$$

but

$$
\rho\left(t_{f}\right)=R^{T}\left(t_{f}\right) \Omega_{12}\left(t_{f}, t_{s}\right) e=R^{T}\left(t_{f}, q=x_{f}^{*}\right) \Omega_{12}\left(t_{f}, t_{s}\right) e=T\left(q=x_{f}^{*}\right) \Omega_{12}\left(t_{f}, t_{s}\right) e,
$$

or

$$
\rho\left(t_{f}\right)=\left(x_{f}^{* T} F_{1}+T\right) \Omega_{12}\left(t_{f}, t_{s}\right) e=0 .
$$

Since $R(\tau) Q^{-1}(\tau)$ is finite ${ }^{8}$ for all $\tau \in\left(t_{s}, t_{f}\right)$, it follows that

$$
V(\tau)=0 \quad \text { for all } \tau \in\left(t_{s}, t_{f}\right) \text {. }
$$

Then, $M\left(t_{s}\right)$ is infinite (see Ref. 2, Section 3.3), and the result follows, since $M\left(t_{s}\right)$ infinite implies a conjugate point (Ref. 6).

Remark 6.1. Lemma 6.2 gives a sufficient condition for $t_{s}$ to be a conjugate point; however, this condition is not necessary, as will be seen in Example 6.2.

Thus far, we have seen that either $t_{s}$ is a conjugate point on $x^{*}$ or there exists an extremal trajectory $x_{\alpha}(\alpha \neq 0, \alpha$ may be unbounded), distinct from $x^{*}$, with associated Lagrange multiplier $\nu_{\alpha}=\nu^{*}$ and going through $x^{*}\left(t_{s}\right)$ at $t=t_{s}$. We have, furthermore, the following lemma.

Lemma 6.3. If two extremal trajectories with the same Lagrange multiplier $\nu$ go through the same point at some time $t_{1}$, then they give the same value to the cost functional between $t_{1}$ and $t_{f}$.

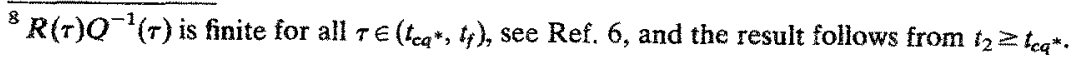


Proof. Let $\bar{x}(t)$ and $x^{*}(t), t \in\left[t_{1}, t_{f}\right]$, be two extremal trajectories such that

$$
\bar{x}\left(t_{1}\right)=x^{*}\left(t_{1}\right) \quad \text { and } \bar{\nu}=\nu^{*} .
$$

The argument used at the beginning of the proof of Lemma 4.2 (see Appendix A) is applicable, with $x_{q}(t)$ replaced by $\bar{x}(t)$, and we have

$$
\Delta J=\frac{1}{2} \Delta x_{f}^{T} F\left(q=\tilde{x}\left(t_{f}\right)\right) \Delta x_{f}-\frac{1}{2} \Delta \lambda^{T}\left(t_{f}\right) \Delta x_{f},
$$

where $\Delta J$ is the difference between the values of the cost functional between $t_{1}$ and $t_{f}$ given by $\bar{x}$ and $x^{*}$,

$$
\Delta x(t) \triangleq \bar{x}(t)-x^{*}(t), \quad \Delta \lambda(t) \triangleq \bar{\lambda}(t)-\lambda^{*}(t),
$$

$\bar{\lambda}(t)$ being the multiplier function associated with $\bar{x}(t)$. But

$$
\begin{gathered}
\bar{\lambda}\left(t_{f}\right)=\left(F_{0}+\bar{\nu} F_{1}\right) \bar{x}\left(t_{f}\right)+\bar{\nu} T, \\
\lambda^{*}\left(t_{f}\right)=\left(F_{0}+\nu^{*} F_{1}\right) x^{*}\left(t_{f}\right)+\nu^{*} T, \\
\bar{\nu}=\nu^{*}
\end{gathered}
$$

imply

$$
\Delta \lambda^{T}\left(t_{f}\right) \Delta x_{f}=\Delta x_{f}^{T}\left(F_{0}+\nu^{*} F_{1}\right) \Delta x_{f}
$$

Then,

$$
\Delta J=0
$$

follows from

$$
F(q)=F_{0}+\nu^{*} F_{1}
$$

Remark 6.2. In the case (iii) mentioned earlier, $\bar{x}(t)$ is the limit of a sequence of trajectories $x_{\alpha}(t)$ as $\alpha$ goes to infinity. For each $x_{\alpha}(t)$, the same argument as in the proof above would give

$$
\Delta J_{\alpha}=-\nu^{*} \psi\left(x_{\alpha}\left(t_{f}\right)\right) \text {. }
$$

Since $x_{\alpha}\left(t_{f}\right)$ is not in $\theta$, the term $-\nu^{*} \psi\left(x_{\alpha}\left(t_{f}\right)\right) \neq 0$ must be added to compensate for $G\left(x_{\alpha}\left(t_{f}\right), v^{*}\right)$ used in $\Delta J_{\alpha}$; see proof of Lemma 4.2 in Appendix A. At the limit,

$$
\Delta J=\lim _{\alpha \rightarrow \infty} \Delta J_{\alpha}=\lim _{\alpha \rightarrow \infty}-\nu^{*} \psi\left(x_{\alpha}\left(t_{f}\right)\right)=0
$$

since

$$
\lim _{\alpha \rightarrow \infty} x_{\alpha}\left(t_{f}\right) \in \theta .
$$


Recapitulating the varous results obtained, it can be seen that the trajectory $x^{*}(t)$ is not globally optimal on $\left[t_{0}, t_{f}\right]$ when $t_{0}<t_{s}$. Indeed, either $t_{s}$ is a conjugate point and $x^{*}$ is not even locally optimal on $\left[t_{0}, t_{f}\right]$, or $t_{s}$ is not a conjugate point and the result follows from Lemmas 6.1,6.3, and 1.1. Then, Corollary 6.1 and Definition 1.1 imply the following theorem.

Theorem 6.1. For the class of problems considered in this section, a Darboux point occurs on an extremal trajectory at $t_{s}$, that is, when the corresponding $S$-matrix becomes infinite.

Note that we have

$$
t_{D}=t_{s}=\sup _{q \in \theta} t_{c q}
$$

Indeed, $t_{c q} \leq t_{s}$ (see Ref. 2) implies

$$
\sup _{q \in \theta} t_{c q} \leq t_{s}
$$

and the result follows from:

$$
t_{D}=\sup _{q \in \theta} t_{c q} \leq \sup _{q \in \theta} t_{c q} \leq t_{s}
$$

(see Section 5). We shall now present two illustrative examples.

Example 6.1. Consider the case when

$$
\begin{gathered}
n=m=2, \quad t_{0}=0, \quad t_{f}=1, \\
A(t)=C(t)=\left[\begin{array}{ll}
0 & 0 \\
0 & 0
\end{array}\right], \quad B(t)=E(t)=\left[\begin{array}{ll}
1 & 0 \\
0 & 1
\end{array}\right], \\
x_{0}=\left[\begin{array}{l}
-0.5 \\
-0.5
\end{array}\right], \quad F_{0}=\left[\begin{array}{ll}
0 & 0 \\
0 & 0
\end{array}\right], \quad F_{1}=\left[\begin{array}{ll}
0 & 0 \\
0 & 2
\end{array}\right], \quad T=[2,0], \quad \psi_{0}=-3
\end{gathered}
$$

in Eqs. (1)-(4) and (20)-(21). This example is a minimum-distance problem from a point to a parabola. An extremal solution is

$$
\begin{gathered}
u^{*}(t)=\left[\begin{array}{c}
\frac{3}{2} \\
\frac{3}{2}
\end{array}\right], \quad x^{*}(t)=\left[\begin{array}{c}
\frac{3}{2}\left(t-\frac{1}{3}\right) \\
\frac{3}{2}\left(t-\frac{1}{3}\right)
\end{array}\right], \quad t \in[0,1], \\
\lambda^{*}(t)=-u^{*}(t), \quad \nu^{*}=-\frac{3}{4} .
\end{gathered}
$$

We have

$$
S(t)=\left[\begin{array}{cc}
0 & 0 \\
0 & -1 /\left(t-\frac{1}{3}\right)
\end{array}\right]
$$


and

$$
M(t, q)=\frac{1}{(1-t)\left[t-\frac{1}{3}+\frac{1}{6}\left(q_{2}+1\right)^{2}\right]}\left[\begin{array}{cc}
t-\frac{1}{3} & \frac{1}{3}\left(q_{2}+1\right) \\
\frac{1}{3}\left(q_{2}+1\right) & t-1+\frac{1}{6}\left(q_{2}+1\right)^{2}
\end{array}\right]
$$

for all

$$
q=\left[\begin{array}{l}
q_{1} \\
q_{2}
\end{array}\right] \in \theta
$$

It follows that

$$
t_{s}=\frac{1}{3} \quad \text { and } t_{c q}=\frac{1}{3}-\frac{1}{6}\left(q_{2}+1\right)^{2} \text {. }
$$

Note that

$$
\sup _{q \in \theta} t_{c q}=\sup _{q_{2} \in(-\infty,+\infty)} t_{c q}=\frac{1}{3}=t_{s} .
$$

The Darboux point

$$
t_{D}=t_{s}=\frac{1}{3}
$$

is of Type-1. Also, note that there exists a trajectory $\bar{x}$, distinct from $x^{*}$, giving the same value to the cost functional between $t_{D}$ and $t_{f}$ as $x^{*}$ between $t_{D}$ and $t_{f}$ (see Fig. 1).

Now, suppose that

$$
x_{0}=\left[\begin{array}{l}
0 \\
0
\end{array}\right]
$$

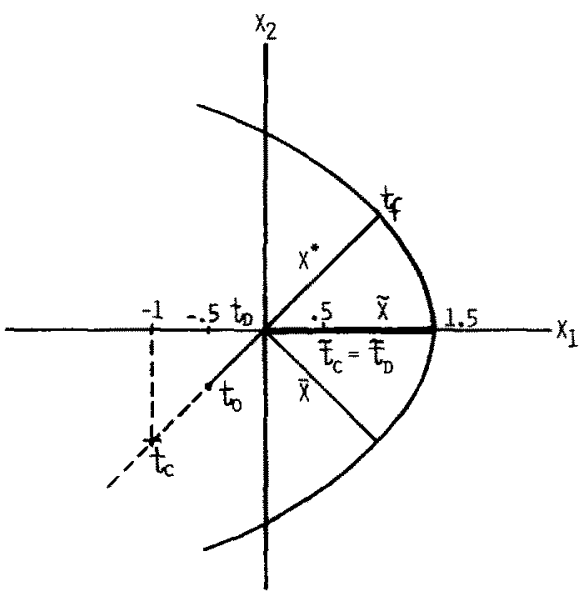

Fig. 1. Darboux points in Example 6.1. 
An extremal solution is

$$
\tilde{u}(t)=\left[\begin{array}{c}
\frac{3}{2} \\
0
\end{array}\right], \quad \tilde{x}(t)=\left[\begin{array}{c}
\frac{3}{2} t \\
0
\end{array}\right], \quad \tilde{\lambda}(t)=-\tilde{u}(t), \quad \tilde{\nu}=-\frac{3}{4} .
$$

We have

$$
\Omega_{22}(\tau, t)=\left[\begin{array}{ll}
1 & 0 \\
0 & 1
\end{array}\right], \quad \Omega_{12}(\tau, t)=-\left[\begin{array}{cc}
\tau-t & 0 \\
0 & \tau-t
\end{array}\right]
$$

Then,

$$
\bar{S}\left(t_{s}, t_{f}\right)=\left[\begin{array}{cc}
1 & 0 \\
0 & 1-\frac{3}{2}\left(1-t_{s}\right)
\end{array}\right],
$$

where $\bar{S}$ is defined in (22). This matrix becomes singular when

$$
t_{s}=\frac{1}{3},
$$

and a unit vector in its null space is

$$
e=\left[\begin{array}{l}
0 \\
1
\end{array}\right]
$$

It follows that

$$
\left(\tilde{x}_{f}^{T} F_{1}+T\right) \Omega_{12}\left(t_{f}, t_{s}\right) e=[2,0]\left[\begin{array}{cc}
-\frac{2}{3} & 0 \\
0 & -\frac{2}{3}
\end{array}\right]\left[\begin{array}{l}
0 \\
1
\end{array}\right]=0 .
$$

It may be shown that the Darbous point

$$
\tilde{t}_{D}=\tilde{t}_{s}=\frac{1}{3}
$$

on $\tilde{x}$ is also a conjugate point, as expected from Lemma 6.2 (see Fig. 1), thus providing an illustration of a $\mathrm{Darboux}$ point which is also a conjugate point.

Example 6.2. Consider the same problem as Example 6.1, but with

$$
F_{1}=\left[\begin{array}{ll}
2 & 0 \\
0 & 2
\end{array}\right], \quad T=[0,0], \quad \psi_{0}=-2, \quad x_{0}=\left[\begin{array}{l}
-0.5 \\
-0.5
\end{array}\right] .
$$

The terminal set $\theta$ is now a circle. The pair $\left(u^{*}(t), x^{*}(t)\right)$ of Example 6.1 is also extremal for this example with the same multipliers $\lambda^{*}(t), \nu^{*}$. The Darboux point is still

$$
t_{D}=t_{s}=\frac{1}{3}
$$

but it is also a conjugate point. We have

$$
\bar{S}\left(t_{s}, t_{f}\right)=\left[\begin{array}{cc}
1-\frac{3}{2}\left(1-t_{s}\right) & 0 \\
0 & 1-\frac{3}{2}\left(1-t_{s}\right)
\end{array}\right] .
$$


When

$$
t_{s}=\frac{1}{3}
$$

$\bar{S}$ becomes identically zero, and any vector $e \in R^{2}$ is in its null space. In particular, choose

$$
e=\left[\begin{array}{l}
1 \\
1
\end{array}\right]
$$

it follows that

$$
\left(x_{f}^{* T} F_{1}+T\right) \Omega_{12}\left(t_{f}, t_{s}\right) e=[2,2]\left[\begin{array}{c}
-\frac{2}{3} \\
-\frac{2}{3}
\end{array}\right]=-\frac{8}{3} \neq 0,
$$

which shows that the condition of Lemma 6.2 is not necessary for $t_{s}$ (the Darboux point) to also be a conjugate point.

\section{Conclusions}

In this paper, characterizations of the Darboux point for particular classes of problems (linear dynamics and quadratic integrand in the cost functional, along with certain analyticity assumptions) have been determined. The Darboux point was shown to be the supremum of a sequence of conjugate points. This approach allows a connection between the conjugate point and the Darboux point on an extremal trajectory, namely, that a conjugate point is a first-order approximation to a Darboux point. When the terminal constraints are limited to a single quadratic constraint, a Darboux point occurs when the Riccati matrix (i.e., the $S$-matrix) associated with the trajectory becomes unbounded. This notable property makes the Darboux point test very simple for this type of problem. To the best of the authors' knowledge, it is the first time that $S \rightarrow \infty$ has been characterized in problems with terminal constraints.

The various results concerning the characterizations of the Darboux point for the classes of problems considered are summarized in Table 1, where $t_{c}$ is the first backward conjugate point, $t_{s}$ is the time when the Riccati matrix becomes infinite, and $t_{c q}, t_{c q}, q, \theta$ are defined in the text.

Table 1. Darboux point characterizations.

\begin{tabular}{lcccc}
\hline & \multicolumn{3}{c}{ Terminal constraints (number and form) } \\
\cline { 2 - 5 } & $\begin{array}{c}0 \leq p \leq n, \\
\text { linear }\end{array}$ & $\begin{array}{c}0 \leq p \leq n-1, \\
\text { arbitrary }\end{array}$ & $\begin{array}{c}p=n-1, \\
\text { arbitrary }\end{array}$ & $\begin{array}{c}p=1, \\
\text { quadratic }\end{array}$ \\
\hline Darboux point occurs at & $t_{c}$ & $\sup _{q \in \theta} \bar{t}_{c q}$ & $\sup _{q \in \theta} t_{c q}$ & $t_{s}$ \\
\hline
\end{tabular}




\section{Appendix A: Proof of Lemmas 2.1 and 4.2}

Proof of Lemma 2.1. ${ }^{9}$ Given $q$, let $\bar{x}(t), t \in\left[t_{0}, t_{f}\right]$, be an admissible trajectory starting at $\bar{x}\left(t_{0}\right)=x_{0}$ and terminating at $\bar{x}\left(t_{f}\right)=q$. It can be shown from (1) that

$$
\begin{aligned}
\bar{J}-J^{*}= & g\left(\bar{x}_{f}\right)-g\left(x_{f}^{*}\right)+\frac{1}{2} \int_{t_{0}}^{t_{f}}\left(\Delta x^{T} C \Delta x+\Delta u^{T} E \Delta u\right) d t \\
& +\int_{t_{0}}^{t_{f}}\left(x^{* T} C \Delta x+u^{* T} E \Delta u\right) d t,
\end{aligned}
$$

where $\widetilde{J}$ and $J^{*}$ are the values of the cost functional given respectively by the trajectories $\bar{x}$ and $x^{*}$,

$$
\Delta x(t) \triangleq \bar{x}(t)-x^{*}(t), \quad \Delta u(t) \triangleq \bar{u}(t)-u^{*}(t),
$$

$\bar{u}(t), t \in\left[t_{0}, t_{f}\right]$, being the control associated with $\bar{x}$. Add the identically zero quantity

$$
\begin{aligned}
0 \equiv & \nu^{* T}\left(\psi\left(\tilde{x}_{f}\right)-\psi\left(x_{f}^{*}\right)\right]+\frac{1}{2} \int_{t_{0}}^{t_{1}} \Delta x^{T} M(t, q)(A \Delta x+B \Delta u-\Delta \dot{x}) d t \\
& +\int_{t_{1}}^{t_{f}}\left[\frac{1}{2} \Delta x^{T} S(t, q)\right. \\
& \left.+\nu^{T} R^{T}(t, q)\right](A \Delta x+B \Delta u-\Delta \dot{x}) d t+\frac{1}{2} \nu^{T}\left[Q\left(t_{1}, q\right)+\int_{t_{1}}^{t_{f}} \dot{Q}(t, q) d t\right] \nu,
\end{aligned}
$$

where $\nu \in R^{p}$ is a constant to be chosen later and $t_{1} \in\left(t_{0}, t_{f}\right)$ is such that the matrix $S(t, q)$ is defined on $\left[t_{1}, t_{f}\right]$. The existence of such $t_{1}$ sufficiently close to $t_{f}$ is implied by the existence of $S\left(t_{f}\right)$. It follows from (15) and (16) that $R(t, q)$ and $Q(t, q)$ are defined on $\left[t_{1}, t_{f}\right]$. Thus, (25) is well defined, since $M(t, q)$ is finite on $\left[t_{0}, t_{1}\right]$. After integrating by parts and using [through necessary conditions (5), (6), (7), and Eq. (11)]

$$
\begin{aligned}
\int_{t_{0}}^{t_{f}}\left(x^{* T} C \Delta x+u^{* T} E \Delta u\right) d t & =-\int_{t_{0}}^{t_{f}}(d / d t)\left(\lambda^{* T} \Delta x\right) d t=-\lambda^{* T}\left(t_{f}\right) \Delta x_{f} \\
& =-\left(d / d x_{f}\right) G\left(x_{f}^{*}, \nu^{*}\right) \Delta x_{f},
\end{aligned}
$$

\footnotetext{
${ }^{9}$ This proof was suggested by similar proofs due to McReynolds (Ref. 7) and Wood (Ref. 8).
} 
we have

$$
\begin{aligned}
\bar{J}-J^{*}= & G\left(\bar{x}_{f}, \nu^{*}\right)-G\left(x_{f}^{*}, \nu^{*}\right)-\left(d / d x_{f}\right) G\left(x_{f}^{*}, \nu^{*}\right) \Delta x_{f}-\frac{1}{2} \Delta x_{1}^{T} M\left(t_{1}, q\right) \Delta x_{1} \\
& -\frac{1}{2} \Delta x_{f}^{T} S\left(t_{f}, q\right) \Delta x_{f} \\
& -\nu^{T} R^{T}\left(t_{f}, q\right) \Delta x_{f}+\frac{1}{2} \Delta x_{1}^{T} S\left(t_{1}, q\right) \Delta x_{1}+\nu^{T} R^{T}\left(t_{1}, q\right) \Delta x_{1} \\
& +\frac{1}{2} \nu^{T} Q\left(t_{1}, q\right) \nu \\
& +\frac{1}{2} \int_{t_{0}}^{t_{1}}\left[\Delta x^{T}\left(C+M A+A^{T} M+\dot{M}\right) \Delta x+\Delta x^{T} M B \Delta u+\Delta u^{T} B^{T} M \Delta x\right. \\
& \left.+\Delta u^{T} E \Delta u\right] d t \\
& +\frac{1}{2} \int_{t_{1}}^{t_{f}}\left[\Delta x^{T}\left(C+S A+A^{T} S+\dot{S}\right) \Delta x+\Delta x^{T} S B \Delta u+\Delta u^{T} B^{T} S \Delta x\right. \\
& +\Delta u^{T} E \Delta u \\
& \left.+2 \nu^{T}\left(R^{T} A+\dot{R}^{T}\right) \Delta x+2 \nu^{T} R^{T} B \Delta u+\nu^{T} \dot{Q} \nu\right] d t
\end{aligned}
$$

where $\Delta x_{1}=\Delta x\left(t_{1}\right)$ and $M, S, R, Q$ in the integrands stand for $M(t, q), S(t, q)$, etc. Then, using (14)-(16), we have

$$
\begin{aligned}
\bar{J}-J^{*}= & G\left(q, \nu^{*}\right)-G\left(x_{f}^{*}, \nu^{*}\right)-\left(d / d x_{f}\right) G\left(x_{f}^{*}, \nu^{*}\right) \Delta x_{f}-\frac{1}{2} \Delta x_{f}^{T} F(q) \Delta x_{f} \\
& -\nu^{T} T^{T}(q) \Delta x_{f} \\
& +\frac{1}{2} \Delta x_{1}^{T} R\left(t_{1}, q\right) Q^{-1}\left(t_{1}, q\right) R^{T}(t, q) \Delta x_{1}+\nu^{T} R^{T}\left(t_{1}, q\right) \Delta x_{1} \\
& +\frac{1}{2} \nu^{T} Q\left(t_{1}, q\right) \nu \\
& +\frac{1}{2} \int_{t_{0}}^{t_{1}}\left[\Delta u+E^{-1} B^{T} M(t, q) \Delta x\right]^{T} E\left[\Delta u+E^{-1} B^{T} M(t, q) \Delta x\right] d t \\
& +\frac{1}{2} \int_{t_{0}}^{t_{f}}\left\{\Delta u+E^{-1} B^{T}[S(t, q) \Delta x+R(t, q) \nu]\right\}^{T} E\{\Delta u \\
& \left.+E^{-1} B^{T}[S(t, q) \Delta x+R(t, q) \nu]\right\} d t .
\end{aligned}
$$

Now, using Assumption (A.4), Eqs. (12)-(13), the condition

$$
\psi(q)=\psi^{*}\left(x_{f}^{*}\right)=0,
$$


which implies

$$
T^{T}(q) \Delta x_{f}=0
$$

and choosing

$$
\nu=-Q^{-1}\left(t_{1}, q\right) R^{T}\left(t_{1}, q\right) \Delta x\left(t_{1}\right),
$$

we are left with the two integrals appearing in the above expression of $J-J^{*}$. Then, Assumption (A.2) implies

$$
J-J^{*} \geq 0 .
$$

Also, the first integral above, between $t_{0}$ and $t_{1}$, cannot be zero; this would imply that

$$
\Delta u(t)=-E^{-1} B^{T} M(t, q) \Delta x(t) \quad \text { a.e. on }\left[t_{0}, t_{1}\right],
$$

which, together with (2), $M(t, q)$ finite on $\left[t_{0}, t_{1}\right]$, and $\Delta x\left(t_{0}\right)=0$ would imply

$$
\Delta x(t) \equiv 0, \quad t \in\left[t_{0}, t_{1}\right],
$$

a contradiction. Hence the result of Lemma 2.1 follows.

Proof of Lemma 4.2. Let

$$
\Delta J \triangleq J_{q}\left[t_{c q}, t_{f}\right]-J^{*}\left[t_{c q}, t_{f}\right]
$$

be the difference between the values of the cost functional given by the trajectories $x_{\mathrm{q}}$ and $x^{*}$ between $t_{\mathrm{cq}}$ and $t_{\mathrm{f}}$, and define

$$
\Delta x(t) \triangleq x_{q}(t)-x^{*}(t), \quad \Delta u(t) \stackrel{\Delta}{=} u_{q}(t)-u^{*}(t),
$$

where $u_{q}(t)$ is the control associated with $x_{q}(t)$. Note that $\Delta x$ and $\Delta u$ are the quantities $y_{\tilde{\alpha}}$ and $v_{\tilde{\alpha}}$ in the proof of Lemma 4.1. since $x_{q}\left(t_{f}\right)=q$ and $x^{*}\left(t_{f}\right)$ are in $\theta$, we can write

$$
\begin{aligned}
\Delta J= & G\left(q, \nu^{*}\right)-G\left(x_{f}^{*}, \nu^{*}\right)+\frac{1}{2} \int_{t_{c q}}^{t_{f}}\left(\Delta x^{T} C \Delta x+\Delta u^{T} E \Delta u\right) d t \\
& +\int_{t_{c q}}^{t_{f}}\left(x^{* T} C \Delta x+u^{* T} E \Delta u\right) d t,
\end{aligned}
$$

where $G\left(x_{f}, \nu^{*}\right)$ was defined in (11).

By construction, $\Delta x(t)$ satisfies the first-order necessary conditions for optimality of (LQP) $)_{q}$ with some multiplier function $\Delta \lambda(t)$. It follows that

and

$$
\Delta u(t)=-E^{-1}(t) B^{T}(t) \Delta \lambda(t)
$$

$$
\begin{aligned}
\Delta x^{T} C \Delta x+\Delta u^{T} E \Delta u & =-(d / d t)\left[\Delta \lambda^{T} \Delta x\right], \\
x^{* T} C \Delta x+u^{* T} E \Delta u & =-(d / d t)\left[\lambda^{* T} \Delta x\right] .
\end{aligned}
$$


Then,

$$
\Delta J=G\left(q, \nu^{*}\right)-G\left(x_{f}^{*}, \nu^{*}\right)-\lambda^{*}\left(t_{f}\right) \Delta x_{f}-\frac{1}{2} \Delta \lambda^{T}\left(t_{f}\right) \Delta x_{f} ;
$$

and, using (7), (11), and (12), we have

$$
\Delta J=\frac{1}{2} \Delta x_{f}^{T} F(q) \Delta x_{f}-\frac{1}{2} \Delta \lambda^{T}\left(t_{f}\right) \Delta x_{f} .
$$

Using

$$
\Delta \lambda\left(t_{f}\right)=F(q) \Delta x\left(t_{f}\right)+T^{T}(q) \nu
$$

for some $\nu$, yields

$$
\Delta J=-\frac{1}{2} \nu^{T} T(q) \Delta x_{f}=-\frac{1}{2} \nu^{T} T(q)\left(q-x_{f}^{*}\right)=0 .
$$

\section{References}

1. Mereau, P., and Powers, W. F., The Darboux Point, Journal of Optimization Theory and Applications, Vol. 17, Nos. 3/4, 1975.

2. Mereau, P., Global Optimality Conditions and the Darboux Point, The University of Michigan, $\mathrm{PhD}$ Thesis, 1974.

3. BLISs, G. A., Lectures on the Calculus of Variations, University of Chicago Press, Chicago, Illinois, 1946.

4. Breakwell, J. V., and Ho, Y. C., On the Conjugate Point Condition for the Control Problem, International Journal of Engineering Science, Vol. 2, pp. $565-579,1965$.

5. Bryson, A. E., and Ho, Y. C., Applied Optimal Control, Blaisdell Publishing Company, Waltham, Massachusetts, 1969.

6. SCHMITENDORF, W. E., and CITRON, S. J., On the Applicability of the Sweep Method to Optimal Control Problems, IEEE Transactions on Automatic Control, Vol. AC-14, pp. 69-72, 1969.

7. MCREYNOLDS, S. R., A Successive Sweep Method for Solving Optimal Program ming Problems, Harvard University, PhD Thesis, 1965.

8. Woon, L. J., Second-Order Optimality Conditions for the Bolza Problem with Interior Point Constraints, Paper presented at the IEEE Decision and Control Conference, San Diego, California, 1973. 